\title{
The Impact of the Parameter Estimation Strategy in the CRS Method
}

Tiago Barros, Rafael Ferrari, Rafael Krummenauer, Renato Lopes and Martin Tygel, University of Campinas

Copyright 2013, SBGf - Sociedade Brasileira de Geofísica.

This paper was prepared for presentation at the $13^{\text {th }}$ International Congress of the Brazilian Geophysical Society, held in Rio de Janeiro, Brazil, August 26-29, 2013.

Contents of this paper were reviewed by the Technical Committee of the $13^{\text {th }}$ International Congress of The Brazilian Geophysical Society and do not necessarily represent any position of the SBGf, its officers or members. Electronic reproduction or storage of any part of this paper for commercial purposes without the written consent of The Brazilian Geophysical Society is prohibited.

\section{Abstract}

The common-reflection surface (CRS) method is a powerful alternative to the traditional commonmidpoint (CMP) stacking, as it enables stacking using more traces than CMP. The CRS method is based on a traveltime that depends on three parameters in the 2D case, and in eight parameters in the 3D case. Traditionally, to keep computational costs low, these parameters are estimated based on a sequence of single-parameter searches, in which each parameter is estimated in turn, while keeping the others fixed. Alternatively, a global optimization method can be used to estimate all parameters simultaneously. In this paper, we analyze the impact of using such a global method. We show that the resulting image may be much improved. We also show that the parameters estimated using the traditional search may not be accurate, and may not result in a large coherence when used in the full CRS traveltime.

\section{Introduction}

The common reflection surface (CRS) method (Herteweck et al., 2007) is a powerful alternative to the common midpoint (CMP) stacking. Both these methods aim to provide a simulated zero offset (ZO) image of the subsurface in time. However, CRS produces images with higher signal-to-noise ratio than those produced by CMP. This improvement is made possible by the fact that CRS uses more traces for stacking than CMP. In fact, the CMP method is based on the normal moveout (NMO) traveltime, which gives the traveltime of a reflection event assuming that all traces have the same midpoint. Thus, the NMO depends only on the offset; however, the number of traces used for stacking is limited by the number of traces sharing a given midpoint. In contrast, the CRS method is based on the CRS traveltime, which depends both on the offset and the midpoint. This enables the use of traces on neighboring CMP gathers ${ }^{1}$ to be used for stacking.

The CRS traveltime depends on more parameters than NMO: three in 2D data, eight in 3D data. On the one hand, this is very advantageous, as this allows for an adequate use of traces in the neighboring CMP gathers for stacking. Also, these parameters carry some information about the geology, and thus may be used for, e.g., tomography (Duveneck, 2004). On the other hand,

\footnotetext{
${ }^{1} \mathrm{~A}$ CMP gather is the set of all traces sharing a given midpoint.
}

estimating several parameters may be costly.

Traditionally, the CRS parameters are estimated using coherence analysis. However, to keep the computational costs low, they are estimated in a sequence of singleparameter searches. First, the parameter associated with the NMO velocity is estimated assuming that the others are zero. This can be done since this first search is performed in a single CMP gather, where the CRS traveltime depends only in the NMO velcoity. Using this parameter, a stacked image is obtained, and the next parameter is estimated on this image. This procedure is repeated sequentially, until all parameters have been estimated. As an alternative, the CRS parameters may be estimated using a global optimization strategy, which searches simultaneously for the combination of all the parameters with the largest coherency (Garabito et al., 2012). This strategy yields better results, at the cost of higher computational complexity.

In this paper, we will study the impact of the global optimization to estimate all parameters simultaneously. We will show that there are mainly two differences between the sequential and the global parameters search strategies. First, the coherence measures in the sequential search are computed in sets of data that are not the same to those used for the CRS traveltime in the global search. As a consequence, these searches use different coherency measures. Second, the quality of the estimate of the parameters in a given stage of the sequential search depends on the results from the previous stages. This latter issue causes an intrinsic propagation of the errors throughout the estimation process, thus degrading the quality of the final stacking result. The impact of the these differences are investigated along this work.

\section{The CRS method}

In this section, we describe the CRS method, which produces a simulated $\mathrm{ZO}$ section from multicoverage data. We will focus on 2D data, although the results of this paper could be extended to the 3D case. We first establish some notation. Thus, let $m_{0}$ be the central point, i.e., the point where the ZO trace is being constructed. As usual, we associate each trace with a source-receiver pair with coordinates $s$ and $r$, respectively. Alternatively, a trace may be identified by the midpoint $m$ and the half-offset $h$ of the source-receiver pair. In this case, the coordinates are related as $s=m-h$ and $r=m+h$. This setting is illustrated in figure 1.

The CRS traveltime relates the traveltime of a reflection that originates at a source in $s$ and is received by a receiver in $r$ with the two-way ZO traveltime $t_{0}$ of the same reflection event. It is written as

$$
t_{\mathrm{CRS}}\left(h, m_{\mathrm{d}}\right)^{2}=\left(t_{0}+a m_{\mathrm{d}}\right)^{2}+b m_{\mathrm{d}}^{2}+c h^{2},
$$




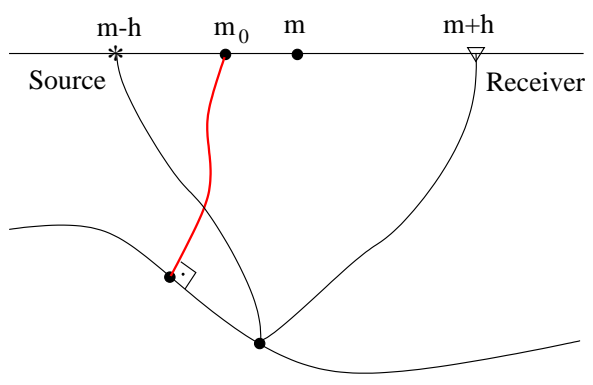

Figure 1: Geometry related to the CRS traveltime, showing the central point, the zero-offset ray in red, and a sourcereceiver pair.

where $m_{\mathrm{d}}=m-m_{0}$ is the trace midpoint displacement, i.e., the difference between the trace midpoint, $m$, and the central point $m_{0}$, the parameters $a$ and $b$ are related, respectively, to the dip and the curvature of the reflector image in the stacked section, and $c$ is related to the normal moveout (NMO) velocity (Jäger et al., 2001). Obviously, $t_{\mathrm{CRS}}\left(h, m_{\mathrm{d}}\right)$ is only an approximation of the actual traveltime, which is only adequate if neither $h$ nor $m_{\mathrm{d}}$ are too big. The selection of the values of $h$ and $m_{\mathrm{d}}$ that are actually used by the CRS method at a given central point is beyond the scope of this paper. Here, we only assume that an appropriate range of these parameters has been provided.

In figure 2, we show the values of the CRS traveltime as a function of the midpoint $m$ and the half-offset $h$ for given values of $m_{0}, t_{0}, a, b$ and $c$. We show only positive values of $h$, but the curve extends symmetrically to negative offsets. In this curve, the black dot corresponds to the central point $m_{0}$ and the time $t_{0}$ of the simulated ZO trace of interest. In blue, we show the CRS traveltime for traces with zero offset, i.e., $t_{\mathrm{CRS}}\left(0, m_{\mathrm{d}}\right)$. The black curves that are almost parallel to this blue curve show the CRS traveltime for traces with common offset, i.e., with constant $h$. In red, we show the CRS traveltime for traces whose midpoint is $m_{0}$, i.e., $t_{\mathrm{CRS}}(h, 0)$. This is a traditional NMO curve. The black curves that are almost parallel to the red curve correspond to the CRS traveltime for traces in other CMP gathers. Using figure 2, we may make several observations that help to explain the CRS method, and that will be important in particular when explaining the strategies to estimate the CRS parameters.

We begin by noting that, as with NMO, the CRS traveltime can be used to estimate the amplitude of the simulated ZO trace located at $m_{0}$ : its sample at time $t_{0}$ is given by the average of the other traces along the corresponding traveltime curve, based on the parameters determined for the given values of $m_{0}$ and $t_{0}$. However, note that the NMO traveltime only depends on $h$; in fact, $t_{\mathrm{NMO}}(h)=t_{\mathrm{CRS}}(h, 0)$. As a consequence, the NMO traveltime can only be used for traces whose midpoint is equal to $m_{0}$, so that $m_{\mathrm{d}}=$ 0 . This is the red curve in figure 2. In contrast, the CRS traveltime enables the use of traces with different midpoints. This explains the better quality of the CRS results.

Based on figure 2, we can think of the CRS stacking as a two-step procedure. First, for each CMP involved, we sum the amplitudes along each of the CMP traveltimes parallel

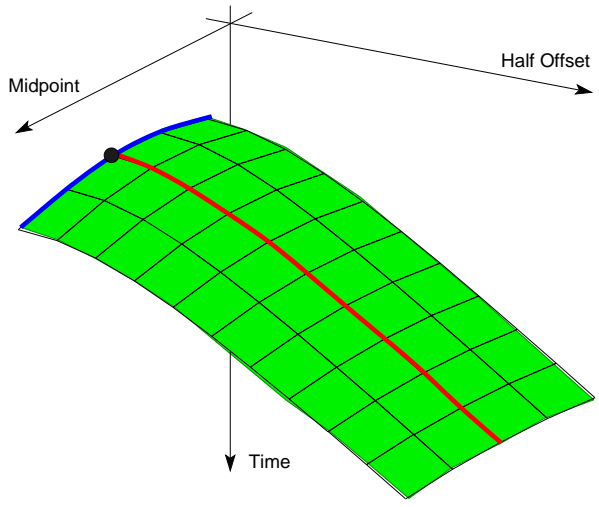

Figure 2: CRS traveltime as a function of $h$ and $m$. The black dot shows the values of $m_{0}$ and $t_{0}$ of interest. The blue curve, and the black curves almost parallel to it, show the CRS traveltime for traces with the same offset. The red curve, and the black curves parallel to it, show the CRS traveltime for traces with a common midpoint.

to the red curve. This is similar to a CMP stack, and creates intermediate ZO traces, one for each CMP involved. Then, we average these $\mathrm{ZO}$ traces along the blue curve shown in the figure to produce the final simulated ZO trace at $m_{0}$ and sample time $t_{0}$. This observation motivates the sequential search strategy of Jäger et al. (2001), to be described in the sequel.

In the two-step description of the CRS stacking, it is important to note that there is a single value of $c$ associated to the central point $m_{0}$ and the ZO time $t_{0}$. Thus, the same value of $c$ is used to generate the several intermediate $Z O$ samples. Obviously, when computing the simulated $Z O$ trace at a different central point, a different value of $c$ may be used, even if the traces with CMP $m_{0}$ are involved in the stacking. This observation will help to explain some differences between the search strategies discussed in this paper.

As with traditional velocity analysis (Taner and Koehler, 1969), the CRS parameters $a, b$ and $c$ in equation 1 are estimated from the data by coherence analysis. The idea is that, for the right parameters, all the traces at time $t_{\mathrm{CRS}}\left(h, m_{\mathrm{d}}\right)$ refer to the same reflection event, so that that these samples should be coherent, or aligned. Ideally, then, for every $t_{0}$ and $m_{0}$ the CRS parameters could be estimated as those that generate a traveltime curve along which the amplitudes of the traces are most coherent. We refer to this strategy as the global CRS search. In keeping with the usual practice, the semblance (Neidell and Taner, 1971) coherence measure will be used in this paper. Note that, for each combination of $a, b$ and $c$, the semblance is computed in a window of data around a traveltime surface as shown in figure 2 .

The problem with testing several combinations of the parameters to determine which one maximizes coherence is complexity. Indeed, if we want to test say 100 values of each of the three parameters in the 2D case, we would have to test one million combinations of parameters. Considering that we would have to compute the semblance for each of these combinations, this procedure is clearly 
unfeasible. In the next section, we review the sequential estimation procedure of (Jäger et al., 2001), which is widely used to estimate the CRS parameters with lower complexity.

\section{Sequential Estimate of the CRS parameters}

In this section, we review the sequential search for the CRS parameters proposed in (Jäger et al., 2001). We will describe this procedure by means of an example, which will better illustrate the process and highlight some of its characteristics. To that end, we consider a model with a single dipping reflector with angle of 10 degrees. We further consider a homogenous overburden with velocity $2,000 \mathrm{~m} / \mathrm{s}$. The synthetic data was generated using 96 channels per shot in a split-spread geometry with offsets $2,500-150-0-150-2,500 \mathrm{~m}$ and $50 \mathrm{~m}$ between the receivers. The distance between the shots is $200 \mathrm{~m}$, which results in 12 traces per CMP. For the considered scenario, the CRS parameters are $c=1 \times 10^{-6} \mathrm{~s}^{2} / \mathrm{m}^{2}$ and $a=1.74 \times 10^{-4} \mathrm{~s} / \mathrm{m}$. Since this is a planar reflector, we know that $b=0$. We focus on the central point $m_{0}=7,650 \mathrm{~m}$ (CMP index 307), at which the reflector depth is $2,618.79 \mathrm{~m}$. In all the results we use the $\mathrm{ZO}$ traveltime $t_{0}=2.579 \mathrm{~s}$, which corresponds to the actual two-way traveltime of the reflection. We further use an aperture of $2,500 \mathrm{~m}$ for the offset and $375 \mathrm{~m}$ for midpoint displacement when performing the searches. We also added random noise to the data, in order to get the average signal-to-noise ratio (SNR) of approximately $-15 \mathrm{~dB}$ along the event. This particular set of parameters was chosen because it reveals several important differences between the sequential search and the global search.

\section{CMP Search}

The first step of the sequential search involves the estimation of $c$, which is related to the NMO velocity. To that end, in order to keep the complexity low, we use a procedure very similar to the traditional velocity analysis in the CMP method: we use only traces whose midpoint is $m=m_{0}$. In this case, $m_{\mathrm{d}}=0$, so that, for these traces,

$$
t_{\mathrm{CRS}}(h, 0)^{2}=t_{0}^{2}+c h^{2} .
$$

In other words, for these traces, $t_{\mathrm{CRS}}\left(h, m_{\mathrm{d}}\right)=t_{\mathrm{NMO}}(h)$, and the traveltime only depends on $c$. The semblance for these traces is then computed for several values of $c$, and we choose the parameter that yields the largest semblance. In the literature, this is called the CMP search. In figure 3, we illustrate the semblance values for several values of $c$ for our example. In this case, the high noise level (SNR $=-15 \mathrm{~dB}$ ) and the low fold (12 traces) degrade the semblance estimation to the point that the coherence peak does not correspond to the correct value of the parameter $c$ : the peak occurs at $c=1.14 \times 10^{-6} \mathrm{~s}^{2} / \mathrm{m}^{2}$ when the correct value is $c=0.97 \times 10^{-6} \mathrm{~s}^{2} / \mathrm{m}^{2}$.

In the global CRS search, we will eventually test parameter combinations having $a=b=0$, and all the values of $c$ used in the CMP search. Further note that when $a=b=0$, then

$$
t_{\mathrm{CRS}}\left(h, m_{\mathrm{d}}\right)^{2}=t_{0}^{2}+c h^{2} .
$$

In this case, $t_{\mathrm{CRS}}\left(h, m_{\mathrm{d}}\right)$ does not depend on $m_{\mathrm{d}}$, which seems to indicate that the maximum in this configuration would be the same as the CMP search. However, in the

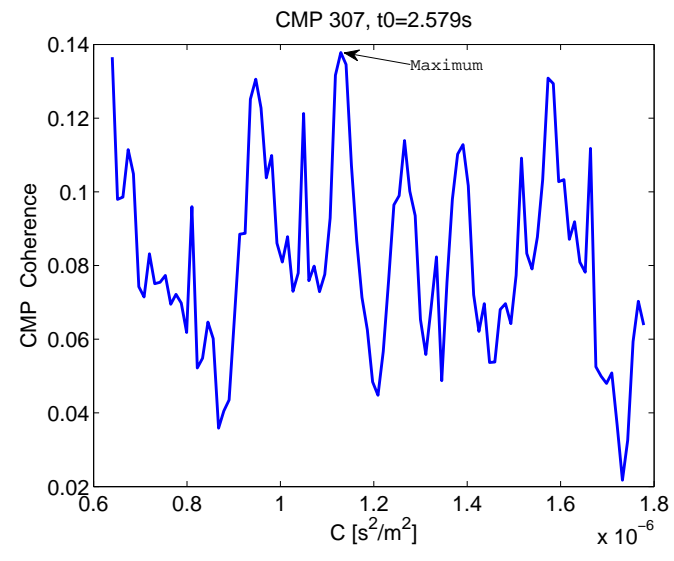

Figure 3: Semblance values for different values of $c$ in the CMP search.

global search, the semblance involves traces with different values of $m_{\mathrm{d}}$, and not only $m_{\mathrm{d}}=0$ as used in the CMP search. Referring to figure 2 , semblance is computed in the whole traveltime surface for the global search, while in the CMP search it is computed only along the red curve.

To illustrate the difference between the semblance in CMP search and in the global search with $a=b=0$, in figure 4 we plot the semblance as a function of $c$ for the global search, computed using $a=b=0$. Compare this result with figure 3 . The smaller value of semblance in the global search can be explained by noting that, due to the receiver dip, we expect that, given an offset, reflections at different midpoints should have different traveltimes. However, when using $a=b=0$, the traveltime only depends on the offset, so in the global search we compute semblance using the same time instant for all traces with a given offset. On the other hand, in the CMP search, we compute semblance using only traces with midpoint $m_{0}$. In other words, when compared to the CMP search, when using $a=b=0$ we compute semblance with more, but incoherent traces, which usually yields a smaller value of semblance.

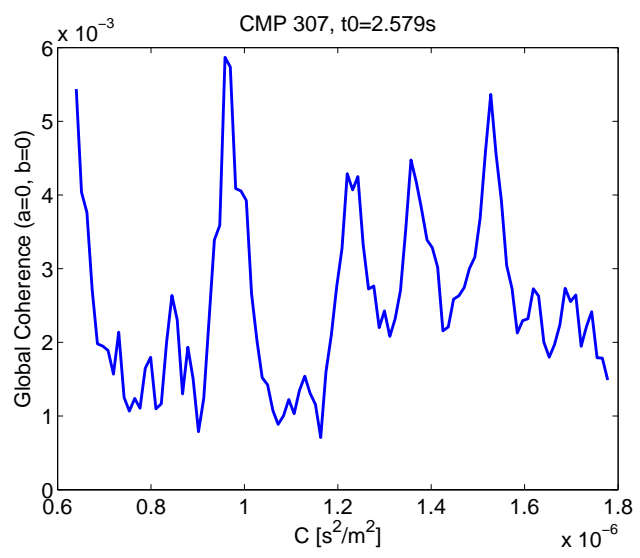

Figure 4: Semblance values for different values of $c$ in a global CRS search using $a=b=0$. 


\section{Angle Search}

The next step in the sequential search is to use the value of $c$, as determined in the CMP search, to generate a simulated $Z O$ section, referred to as the CMP ZO section. The procedure here is similar to the traditional CMP stacking. From the point of view of the search, what is interesting is that the traces in the ZO section all have $h=0$. Thus, for these traces, the CRS traveltime simplifies to

$$
t_{\mathrm{CRS}}\left(m_{\mathrm{d}}, 0\right)^{2}=\left(t_{0}+a m_{\mathrm{d}}\right)^{2}+b m_{\mathrm{d}}^{2} .
$$

To further decrease complexity, we first assume ${ }^{2}$ that $b=0$, so that the traveltime reduces to

$$
t_{\mathrm{CRS}}\left(m_{\mathrm{d}}, 0\right)=t_{0}+a m_{\mathrm{d}} .
$$

This is the equation of a line with angular coefficient $a$.

As usual, the search for $a$ proceeds by coherence analysis, computed, for each value of $a$, on a window on the ZO section around the traveltime in equation 5 . This window is illustrated in figure 5(a). We refer to this step as the angle search. Here, there are two subtle points to be noted:

- First, the search for $a$ is, to some extent, unrelated to the actual CRS traveltime. In fact, as we mentioned when discussing figure 2, in the global CRS search the traveltime surface uses the same value of $c$ for all midpoints. On the other hand, each point in the CMP ZO section was probably obtained with different values of $c$.

- Second, the midpoint aperture used in the angle and curvature searches is generally small, so that the coherence is computed with few traces. This may result in coherence measures that are too optimistic. (Note that the semblance computed from a single trace is always one.) Further, considering the semblance equation

$$
S=\frac{\sum_{i \in W}\left|\sum_{j \in N} u_{i, j}\right|^{2}}{N \sum_{i \in W} \sum_{j \in N}\left|u_{i, j}\right|^{2}},
$$

where $W$ is the length of the time window, $N$ is the number of traces and $u_{i, j}$ is the windowed data in the corresponding domain, note that the denominator of the semblance computed in the angle search is related to the sum of the squares of the samples in the window in figure 5(a). All these samples are the result of stacking some CMP gather. On the other hand, as seen in figure 2 , the semblance computed using the actual CRS traveltime would involve the sum of the squares of several samples in each CMP, before stacking. As the sum of the squares is different than the square of the sum, those two sums can be quite different, even if the same value of $c$ is used to obtain the samples in the CMP ZO section.

\section{Curvature Search and Stacking}

The final step in the sequential search is to estimate $b$. This step is referred to as the curvature search, and it uses the

\footnotetext{
${ }^{2}$ To see a justification for this and other steps in the sequential search, the reader is referred to (Jäger et al., 2001).
}

same ZO section as in the angle search. We also use the value of $a$ obtained in the angle search, so that now the traveltime

$$
t_{\mathrm{CRS}}\left(m_{\mathrm{d}}, 0\right)^{2}=\left(t_{0}+a m_{\mathrm{d}}\right)^{2}+b m_{\mathrm{d}}^{2}
$$

depends only on $b$. We then use this traveltime to estimate $b$ by coherence analysis, following steps similar to those in the angle search. The window used for computing semblance is similar to the one shown in figure $5(\mathrm{a})$; the difference is that its angle will be fixed, and the window will have a parabolic shape controlled by $b$. The subtleties mentioned in the angle search also apply here.

At this point, we can perform stacking in two ways:

- First, we may refine the ZO section. To that end, we perform stacking directly on the ZO image obtained after the CMP search. This stacking is performed along the traveltime given by equation 7, using the values of $a$ and $b$ obtained in the angle and curvature searches. The result is shown in figure $5(b)$. We refer to this method as the curvature stacking.

- Alternatively, we may use the pre-stack data, stacking along the CRS traveltime in equation 1 using the values of $a, b$ and $c$ obtained by the sequential search. The result is shown in figure $6(a)$.

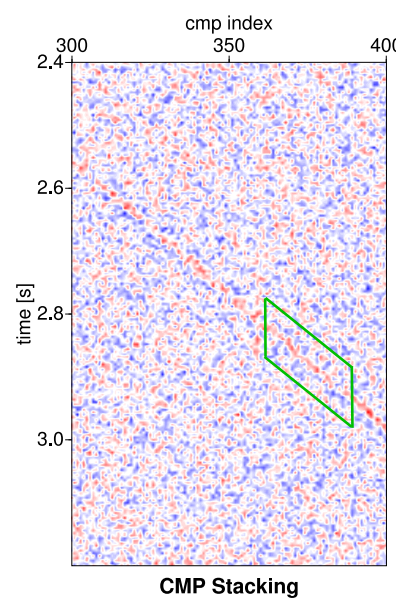

(a)

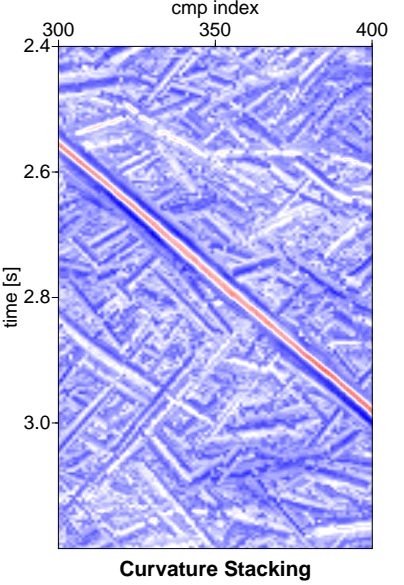

(b)
Figure 5: (a) Windowing the ZO section to compute the semblance for a given $t_{0}$ and a given $a$. We use $b=0$ in this search. (b) Stacked section obtained by refining the ZO section from the CMP mode.

Comparing the results of figures $5(\mathrm{~b})$ and $6(\mathrm{a})$, we see that stacking with the CRS traveltime using the parameters estimated by the sequential search leads to rather poor results in this example. This is explained, to a large extent, by the poor estimate of $c$ in the CMP search: if a wrong value of $c$ is used to produce a CRS traveltime surface, the traces at all midpoints will have a wrong $c$. Thus, as discussed in the two-step description of CRS stacking, all intermediate CMP stacks will be performed with the wrong $c$. In other words, the CRS stack cannot recover from this wrong estimate of $c$, which results in the poor 
stacking results in figure 6(a). On the other hand, in many cases, the estimate may be very poor at a given $t_{0}$ and $m_{0}$, while it may be reasonable at neighboring central points. Thus, the CMP ZO section may have a few points along the reflector image that were well stacked. In the curvature stacking, since we work on the ZO section, the sample at $t_{0}$ and $m_{0}$ will benefit from the good results of its neighbors, explaining why the reflector appears more clearly in figure $5(\mathrm{~b})$.

Also, the CRS stack in figure 6(a) seems to have fewer artifacts in regions without reflectors. These artifacts, known as worms (Gamboa, 2007), are a well-known disadvantage of the CRS method. As we will see, the global search seems to further mitigate the worms. The reasons for that are still not fully understood, and are the subject of current investigation.

\section{Global Search}

Probably the most obvious drawback of the sequential search is that it does not find the optimal estimates of the parameters, in the sense of maximizing the coherence for the CRS traveltime in equation 1. As we have seen in the last section, this may have some serious consequences, both in the values of the parameters themselves and on the quality of the result. To circumvent these problems, while keeping a reasonable computational complexity, Garabito et al. (2012) proposed a global optimization strategy based on the very-fast simulated annealing (VFSA) algorithm (Sen and Stoffa, 2013) to estimate all the CRS parameters in a single search procedure. The details of the method are beyond the scope of this paper. However, since the VFSA may converge to local optima (Sen and Stoffa, 1995), we employed an exhaustive search in the simulations presented here to guarantee the convergence to the global optimum.

To assess the impact of the global search approach, we consider again the synthetic example used in the previous section. In figures $6(a)$ and $6(b)$, we show a comparison of the stacked images obtained with the parameters from the sequential and the global searches, respectively.

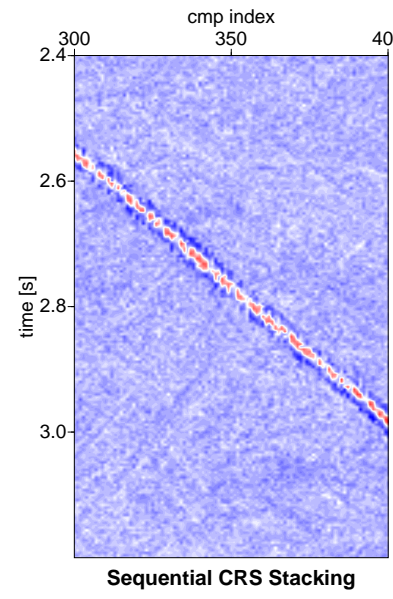

(a)

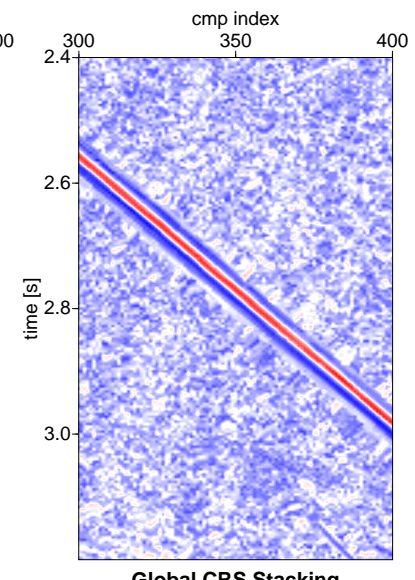

Global CRS Stacking

(b)
Figure 6: Stacked sections obtained from the CRS traveltime, using the sequential (a) and the global (b) search.
To explain the differences between the results, first note that if one of the stages of the sequential search procedure fails to find the optimum parameter, the CRS traveltime will not be able to stack coherently the traces, resulting in a poor image. More specifically, the estimation of $a$ and $b$ relies on the stacked section from the CMP gather, which depends exclusively on the estimated $c$. In figure 7 we show the NMO velocity estimates for the sequential (in red) and the global (in black) searches along the event. Note that the velocities obtained with the CMP search differ greatly from the globally optimal velocities. The large errors in the velocity estimates explain the noisy stacked section of the sequential CRS.

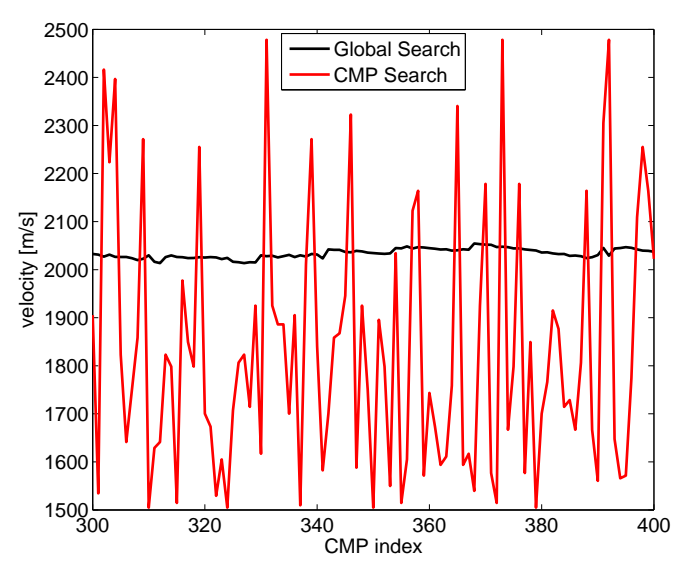

Figure 7: Semblance values for different values of $c$ in the CMP search.

Let us further investigate the global search from the point of view of coherence. Specifically, we focus again on CMP index 307 and $t_{0}=2.579 \mathrm{~s}$. In figure 8 , we show the semblance for different values of the parameters $a$ and $c$, while making $b=0$. We use $b=0$ (the correct value of the parameter) to make the visualization easier. However, plots of semblance varying the three parameters have been shown in (Garabito et al., 2012). In this figure, we also show the estimates obtained by the sequential search and the global search. As we can see, the global search does a very good job at estimating both parameters in this case, while the sequential search fails to produce a good estimate of $c$. (As we mentioned before, the estimate of $a$ in the sequential search may be good, as long as the estimate of $c$ is reasonably accurate in enough points inside the window where the coherence for $a$ is computed.)

As discussed earlier, the bad estimate of $c$ in sequential search is due to the noise that degrades the semblance evaluation, which leads to a coherence peak at a wrong value of $c$. The same is not observed in the coherence of the global search in figure 8: the semblance has a well defined peak at the optimal set of parameters of the CRS traveltime. This is because the semblance in the global search is evaluated using a much larger dataset than in the CMP search. In our example, while the CMP search uses samples from 12 traces, the global search uses samples from 372 traces (the spacing between CMP's is $25 \mathrm{~m}$, there are 12 traces per CMP and $-375 \leq m_{\mathrm{d}} \leq 375 \mathrm{~m}$ ). This means that, for a given SNR, the global semblance is more 


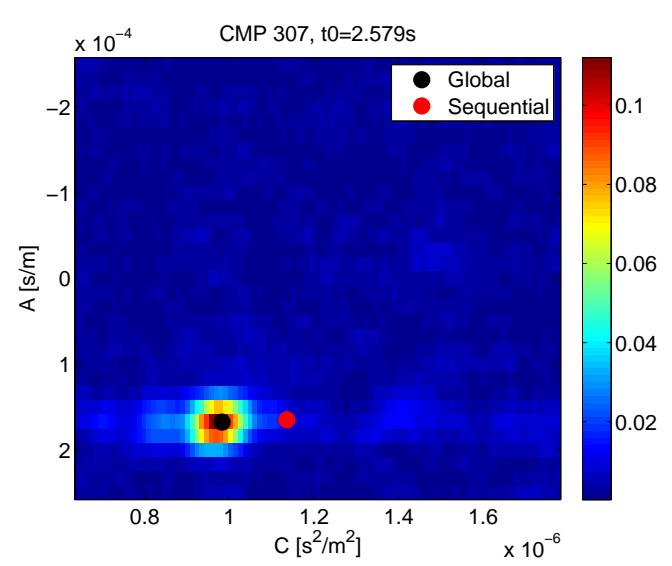

Figure 8: Semblance versus $a$ and $c$. The curvature remains fixed at its correct value, $b=0$. We also show the parameters estimated in the sequential search $\left(a=1.65 \times 10^{-4} \mathrm{~s} / \mathrm{m}\right.$ and $\left.c=1.14 \times 10^{-6} \mathrm{~s}^{2} / \mathrm{m}^{2}\right)$ and in the global search $\left(a=1.68 \times 10^{-4} \mathrm{~s} / \mathrm{m}\right.$ and $\left.c=0.98 \times 10^{-6} \mathrm{~s}^{2} / \mathrm{m}^{2}\right)$.

\section{accurate than the semblance of the CMP search.}

For a sufficiently low SNR, an event that cannot be seen in the CMP can eventually be identified in the global coherence. In figure 9 we show an example that confirms this statement. We processed the same synthetic data but with a lower SNR, more precisely $-23 \mathrm{~dB}$, and the resulting CRS stackings of the sequential and global searches are depicted in figures 9(a) and 9(b) respectively.

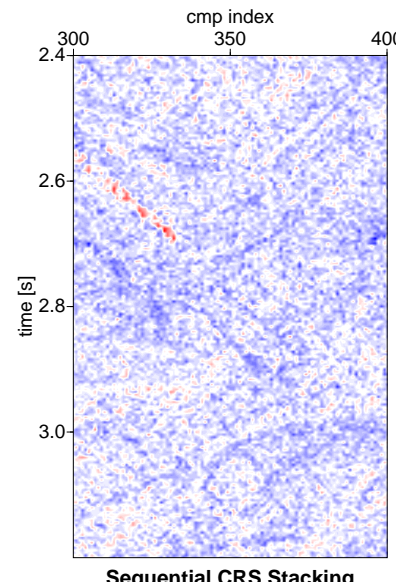

(a)

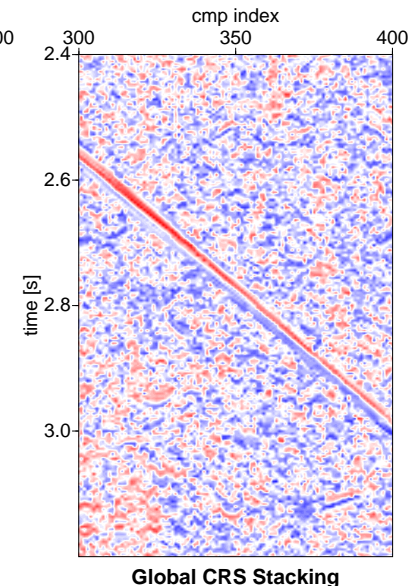

(b)
Figure 9: Stacked sections obtained from the CRS traveltime, using the sequential (a) and the global (b) search for $\mathrm{SNR}=-23 \mathrm{~dB}$.

This scenario emphasizes the performance difference between the two strategies: due to the high noise level, the sequential search is unable to find the correct values of the parameters of the CRS traveltime in such a way that the traces are not coherently stacked and the event is missed. On the other hand, in the global search stacked section, we can still identify the reflector with reasonable quality.

\section{Conclusions}

We discussed the impact of the global optimization to estimate all the CRS traveltime parameters simultaneously, and highlighted the advantages of the global search when compared to the standard estimation strategy for CRS, which estimates these parameters with sequential oneparameter searches in different domains. The first step of the sequential search, which estimates the parameter related to the stacking velocity, is performed in CMP gathers, where the coherence is computed in a single midpoint. This is in contrast to the global search, where a large number of traces with different midpoints is used, resulting in coherence values that are less affected by the noise. We also showed that using a wrong value of $c$, such as those produced by the CMP search sometimes, for stacking with the CRS traveltime may produce bad results. This advantage of the global search is particularly important in situations with a lot of noise and/or a small fold, in which the CMP search may fail to estimate $c$. We showed that the angle and curvature searches may produce good estimates of $a$ and $b$ even when the estimates of $c$ are poor, as long as enough points have good velocity estimation, even though their coherence measure is to a large extent very different from that of the global search. Finally, the results in this paper seem to indicate that using the CRS traveltime for stacking seems to produce fewer worms in the result. This claim needs much more investigation, and is still the topic of current research.

\section{Acknowdegments}

The authors thank FAPESP, CNPq and INCT-GP/CNPQ (www.inct-gp.org) for the financial support.

\section{References}

Duveneck, E., 2004, Tomographic determination of seismic velocity models with kinematic wavefield attributes: Doctoral Thesis, Universität Karlsruhe (TH).

Gamboa, F., 2007, Aplicações do método de Superfície Comum de Reflexão (CRS) ao processamento sísmico: Tese de Doutorado em Ciências e Engenharia de Petróleo, Universidade Estadual de Campinas.

Garabito, G., P. L. Stoffa, L. Lucena, and J. C. Cruz, 2012, Part i-crs stack: Global optimization of the 2d crsattributes: Journal of Applied Geophysics.

Herteweck, T., J. Schleicher, and J. Mann, 2007, Data stacking beyond $\mathrm{cmp}$ : The Leading Edge, 70, 818-827.

Jäger, R., J. Mann, G. Höcht, and P. Hubral, 2001, Common-reflection-surface stack: image and attributes: Geophysics, 66, 97-109.

Neidell, N., and M. Taner, 1971, Semblance and other coherency measures for multichannel data: Geophysics, 36, 482-497.

Sen, M., and P. Stoffa, 1995, Global Optimization Methods in Geophysical Inversion, 2nd ed.: Elsevier, volume 4 of Advances in Exploration Geophysics.

Sen, M. K., and P. L. Stoffa, 2013, Global optimization methods in geophysical inversion: Cambridge University Press.

Taner, M., and F. Koehler, 1969, Velocity spectra digital computer derivation and applications of velocity functions: Geophysics, 34, 859-881. 